\title{
International Year of the Salmon Survey of Fisheries and Oceans Canada Salmon Staff Identifies Collaborative Opportunities and Possible Next Steps
}

\author{
James R. Irvine $^{1}$, Scott Akenhead ${ }^{1}$, and J. Scott Carley $^{2}$ \\ ${ }^{1}$ Fisheries and Oceans Canada, Pacific Biological Station, 3190 Hammond Bay Road, Nanaimo, BC V9T 6N7 \\ Canada \\ ${ }^{2}$ Organomics Consulting, 602 22nd Avenue East, Seattle, Washington 98112 USA
}

Keywords: International Year of Salmon, Survey, Collaboration, Knowledge Sharing, Knowledge Discovery, IYS

Preliminary analysis of results from a 2017 International Year of the Salmon (IYS) survey confirmed collaborative opportunities exist that should allow Fisheries and Oceans Canada (DFO) to better understand and manage salmon populations.

Using Survey Monkey, we asked 351 staff from across the country who work on Pacific or Atlantic salmon if they saw potential for collaboration on 37 topics within six IYS themes (i.e., Status of Salmon, Salmon in a Changing Salmosphere, New Frontiers, Human Dimensions (connecting salmon with people), Information Systems, and Salmon Outreach and Communication). Response rates among six job types (resource managers, line managers, scientists, science biologists, technical staff, hatchery staff) and seven regions (Pacific (British Columbia/Yukon), Central and Arctic, Ottawa, Gulf, Maritimes, Quebec, Newfoundland and Labrador) were similar ( 40\%); 124 surveys nationally were usable for analyses including 90 from the Pacific Region.

Responses to the query of potential enhanced collaboration were scored as (score; figure label in brackets): no or not applicable ( 0 ; NA); yes but unlikely at present (1; pending); yes and I have activity that would benefit ( 2 ; need); yes and I can help others (3; offer); and yes collaboration is vital to my work and should be high priority to DFO (4; critical). Various analyses were applied to the 124 by 37 matrix of answers, including heat maps factored by job types within regions (see the example, Fig. 1) and scoring the topics as weighted means for the answers (weights 0 to 4). Here we present preliminary findings from the analysis of results from the Pacific Region, which had by far the largest sample size $(n=90)$.

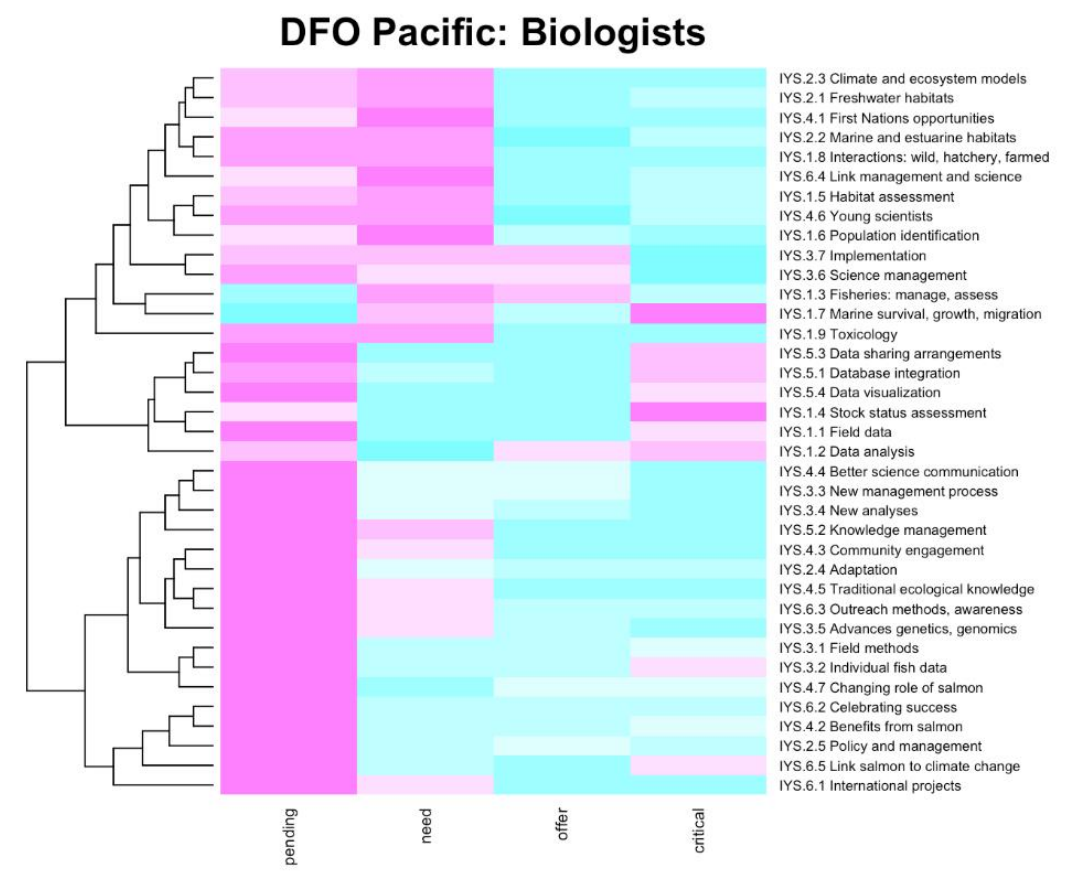

Fig. 1. Cluster analysis of responses to opportunities for collaboration for 37 topics related to salmon. The "no" answers were deleted in this example to emphasize clusters where collaboration was favored. Clustering is by the order of topics, the columns correspond to the available answers (pending, need, offer, and critical). Highest scored topics indicated by dark red purple. 
Table 1. The most important IYS themes and topics from a collaborative perspective identified in a survey of DFO staff from the Pacific Region and corresponding job types for whom these themes and topics were most important.

\begin{tabular}{|c|c|c|}
\hline Themes & & Job Types \\
\hline 1. & Salmon Status & Hatchery Staff, Scientists \\
\hline 2. & Information Systems & Technical Staff, Biologists \\
\hline 3. & Outreach and Communications & Scientists, Hatchery Staff, Managers \\
\hline Topics & & Job Types \\
\hline 1. & Field Data & Hatchery Staff, Resource Managers \\
\hline 2. & Data Analysis & Biologists, Technical Staff \\
\hline 3. & Science Communication & Managers, Biologists, Scientists, Hatchery Staff \\
\hline
\end{tabular}

Salmon Status, Information Systems, and Outreach and Communication were the three most important IYS themes identified from a collaborative perspective while Field Data Collection, Data Analysis, and Science Communication were the three highest ranked topics (Table 1). These results suggest a need for improved collaboration in the flow of information at multiple stages with respect to salmon assessment, management and research (Fig. 2). For instance:

- Various types of Field Data and other information are collected - technicians, biologists, and resource managers identified this as the most important topic for collaboration.

- The information collected needs to be stored in Information Systems and analysed. In highlighting the potential benefits of collaborating on Data Analysis and the Information Systems theme, technical staff and biologists appear to be asking for collaboration support.

- These data are important in determining stock status, and this information needs to be communicated outwards, as identified by managers, biologists and scientists, and hatchery staff.
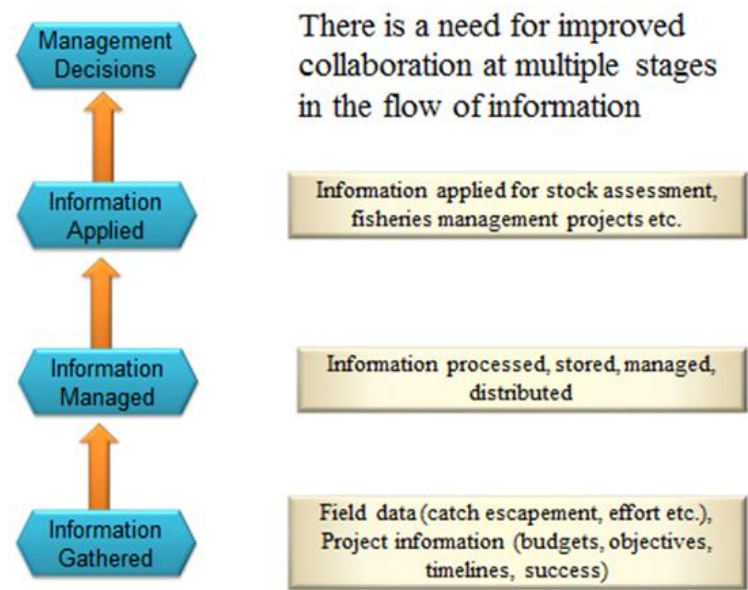

Fig. 2. Information flow for salmon management, from gathering information to making decisions. Our analysis of the survey data indicated a need for improved collaboration at multiple stages in this flow of information.

How then to improve the flow of information?

A working prototype of new web-based technology for knowledge sharing (Fig. 3) was applied to a subset of data gathered during our surveys. RoundTable ${ }^{\mathrm{tm}}$ can deal with diverse kinds of data as a network where there are many nodes of many different types (with extensive properties), which are richly linked together, and then crosslinked to ideas (Fig. 4). Further, the nodes representing ideas are linked into a semantic web. The technology has an appropriate tagline: Connect the dots. All the dots (https://www.roundtable.eco/). Users can view this diversity and complexity (and realism) as tables, graphs or potentially many other interactive data visualizations to support their discovery and sharing of knowledge. 


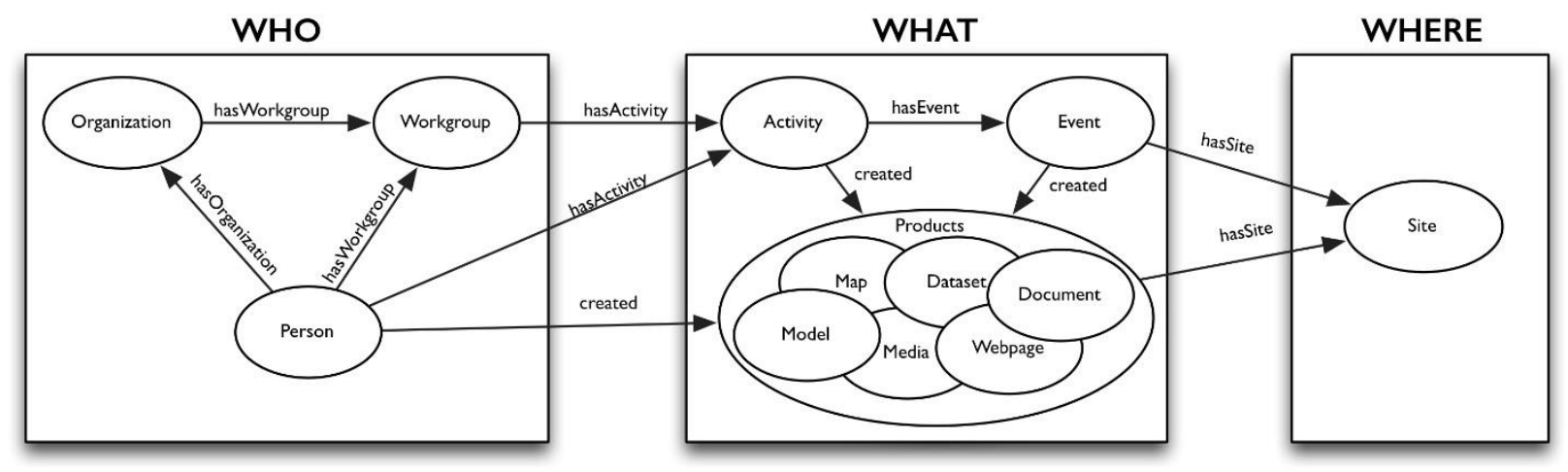

Fig. 3. Example of how information can flow from who (i.e., named individuals, groups, and organizations) to what (i.e., activities (projects), events, products) to where (locations or sites). These links can be customized for each analysis.

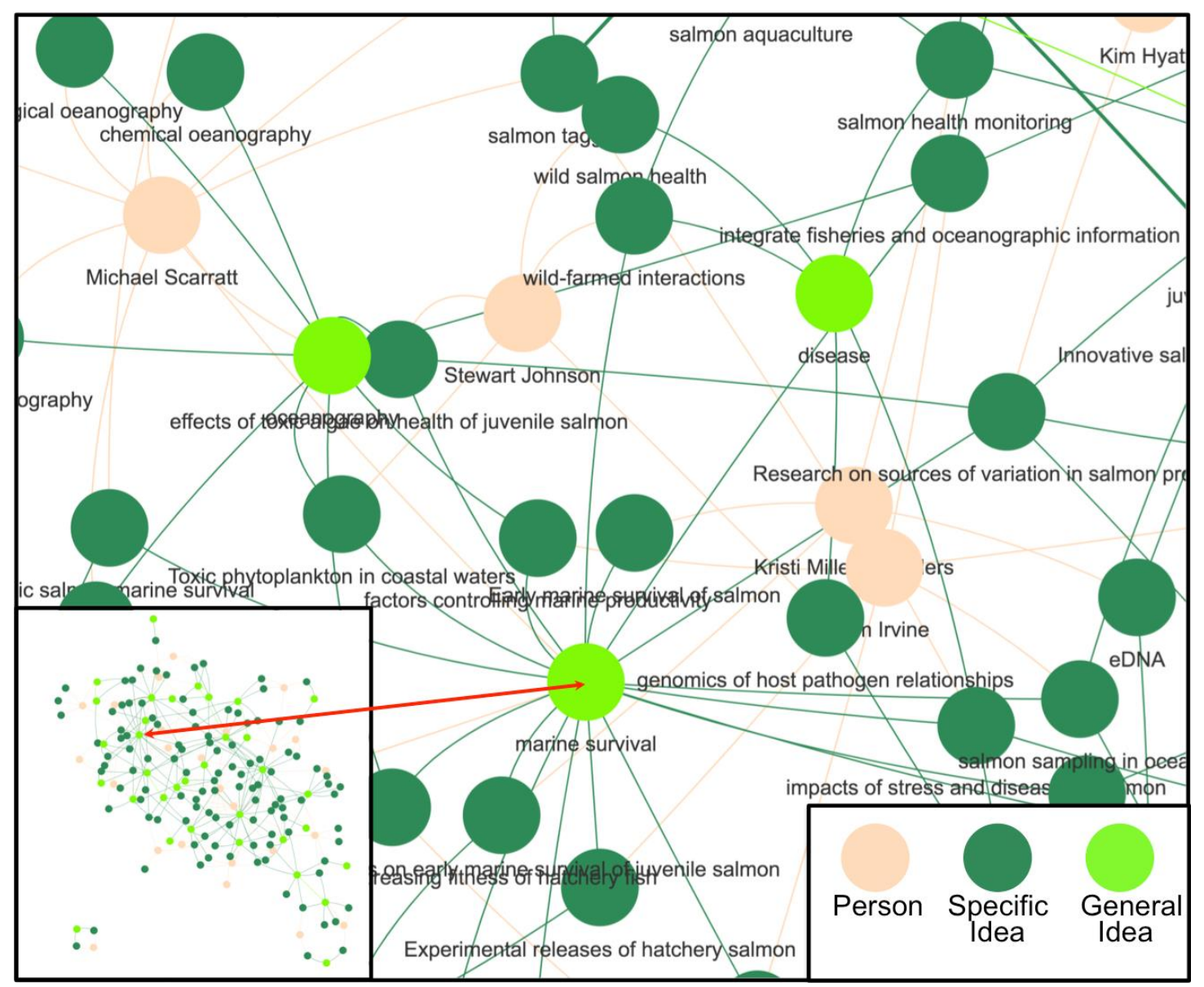

Fig. 4. Results from preliminary analysis of survey data displayed as a Knowledge Graph in bottom left hand corner and a blown-up fragment of it (main figure) showing Persons linked to Specific Ideas they proffered, with those Ideas further linked by more General Ideas. Thus people (activities etc.) can be linked by chains of ideas.

Next steps will hopefully include using RoundTable ${ }^{\mathrm{tm}}$ or similar software to manage a more complete listing of salmon activities (projects). The approach could be extended to others outside DFO who work on salmon, potentially expanding the emerging "DFO Salmon Network" to one with national or international membership. It is our contention that identifying connections among activities and people should result in new and improved partnerships that will improve our ability to manage salmon in an uncertain future. 